\title{
IndicNLPSuite: Monolingual Corpora, Evaluation Benchmarks and Pre-trained Multilingual Language Models for Indian Languages
}

\author{
Divyanshu Kakwani ${ }^{1}$, Anoop Kunchukuttan ${ }^{2 *}$, Satish Golla ${ }^{3 *}$, Gokul N.C. ${ }^{4}$, \\ Avik Bhattacharyya ${ }^{5}$, Mitesh M. Khapra ${ }^{6}$, Pratyush Kumar ${ }^{7}$ \\ Robert Bosch Centre for Data Science and AI, IIT Madras ${ }^{1,6,7}$, Microsoft India ${ }^{2}$, AI4Bharat ${ }^{3,4,5}$ \\ \{divk, miteshk, pratyush\}@cse.iitm.ac.in ${ }^{1,6,7}$, \\ ankunchu@microsoft. com $^{2}$, gokulnc@ai4bharat. org ${ }^{4}$, \\ \{gsatishkumaryadav, avikbhattacharyya. $2 \mathrm{k}$ \}@gmail. $\mathrm{com}^{3,5}$
}

\begin{abstract}
In this paper, we introduce NLP resources for 11 major Indian languages from two major language families. These resources include: (a) large-scale sentence-level monolingual corpora, (b) pre-trained word embeddings, (c) pre-trained language models, and (d) multiple NLU evaluation datasets (IndicGLUE benchmark). The monolingual corpora contains a total of 8.8 billion tokens across all 11 languages and Indian English, primarily sourced from news crawls. The word embeddings are based on FastText, hence suitable for handling morphological complexity of Indian languages. The pre-trained language models are based on the compact ALBERT model. Lastly, we compile the Indic GLUE benchmark for Indian language NLU. To this end, we create datasets for the following tasks: Article Genre Classification, Headline Prediction, Wikipedia Section-Title Prediction, Cloze-style Multiple choice QA, Winograd NLI and COPA. We also include publicly available datasets for some Indic languages for tasks like Named Entity Recognition, Cross-lingual Sentence Retrieval, Paraphrase detection, etc. Our embeddings are competitive or better than existing pre-trained embeddings on multiple tasks. We hope that the availability of the dataset will accelerate Indic NLP research which has the potential to impact more than a billion people. It can also help the community in evaluating advances in NLP over a more diverse pool of languages. The data and models are available at https: //indicnlp.ai4bharat.org.
\end{abstract}

\section{Introduction}

Distributional representations are the corner stone of modern NLP, which have led to significant advances in many NLP tasks like text classification, NER, sentiment analysis, MT, QA, NLI, etc. Particularly, word embeddings (Mikolov

Volunteer effort for the AI4Bharat project et al., 2013b), contextualized word embeddings (Peters et al., 2018), and language models (Devlin et al., 2019) can model syntactic/semantic relations between words and reduce feature engineering. These pre-trained models are useful for initialization and/or transfer learning for NLP tasks. They are also useful for learning multilingual embeddings which enable cross-lingual transfer. Pretrained models are typically learned from large, diverse monolingual corpora. The quality of embeddings is impacted by the size of the monolingual corpora (Mikolov et al., 2013a; Bojanowski et al., 2017), a resource not widely available for many major languages.

In particular, Indic languages, widely spoken by more than a billion speakers, lack large, publicly available monolingual corpora. They include 8 out of top 20 most spoken languages and $\sim 30$ languages with more than a million speakers. There is also a growing population of users consuming Indian language content (print, digital, government and businesses). Further, Indic languages are very diverse, spanning 4 major language families. The Indo-Aryan and Dravidian languages are spoken by $96 \%$ of the population in India. The other families are diverse, but the speaker population is relatively small. Almost all Indian languages have SOV word order and are morphologically rich. The language families have also interacted over a long period of time leading to significant convergence in linguistic features; hence, the Indian subcontinent is referred to as a linguistic area (Emeneau, 1956). Indic languages are thus of great interest and importance for NLP research.

Unfortunately, the progress on Indic NLP has been constrained by the unavailability of large scale monolingual corpora and evaluation benchmarks. The former allows the development of pretrained language models and deep contextualised word embeddings which have become drivers of 
modern NLP. The latter allows systematic evaluation across a wide variety of tasks to check the efficacy of new models. With the hope of accelerating Indic NLP research, we address the creation of (i) large, general-domain monolingual corpora for multiple Indian languages, (ii) word embeddings and multilingual language models trained on this corpora, and (iii) an evaluation benchmark comprising of various NLU tasks.

Our monolingual corpora, collectively referred to as IndicCorp, contains a total of 8.8 billion tokens across 11 major Indian languages and English. The articles in IndicCorp are primarily sourced from news crawls. Using Indic Corp, we first train and evaluate word embeddings for each of the 11 languages. Given the morphological richness of Indian languages we train FastText word embeddings which are known to be more effective for such languages. To evaluate these embeddings we curate a benchmark comprising of word similarity and analogy tasks (Akhtar et al., 2017; Grave et al., 2018), text classification tasks, sentence classification tasks (Akhtar et al., 2016; Mukku and Mamidi, 2017), and bilingual lexicon induction tasks. On most tasks, the word embeddings trained on our IndicCorp outperform similar embeddings trained on existing corpora for Indian languages.

Next, we train multilingual language models for these 11 languages using the ALBERT model (Lan et al., 2020). We chose ALBERT as the base model as it is very compact and hence easier to use in downstream tasks. To evaluate these pretrained language models, we create an NLU benchmark comprising of the following tasks: article genre classification, headline prediction, named entity recognition, Wikipedia section-title prediction, cloze-style multiple choice QA, natural language inference, paraphrase detection, sentiment analysis, discourse mode classification, and crosslingual sentence retrieval. We collectively refer to this benchmark as IndicGLUE and it is a collection of (i) existing Indian language datasets for some tasks, (ii) manual translations of some English datasets into Indian languages done as a part of this work, and (iii) new datasets that were created semi-automatically for all major Indian languages as a part of this work. These new datasets were created using external metadata (such as website/Wikipedia structure) resulting in more complex NLU tasks. Across all these tasks, we show that our embeddings are competitive or better than existing pre-trained multilingual embeddings such as mBERT (Devlin et al., 2019) and XLM-R (Conneau et al., 2020). We hope that these embeddings and evaluations benchmarks will not only be useful in driving NLP research on Indic languages, but will also help in evaluating advances in NLP over a more diverse set of languages.

In summary, this paper introduces IndicNLPSuite containing the following resources for Indic NLP which will be made publicly available:

- IndicCorp: Large sentence-level monolingual corpora for 11 languages from two language families (Indo-Aryan branch and Dravidian) and Indian English with an average 9-fold increase in size over OSCAR.

- IndicGLUE: An evaluation benchmark containing a variety of NLU tasks.

- IndicFT and IndicBERT: FastText-based word embeddings (11 languages) and ALBERT-based language models (12 languages) trained on IndicCorp. The IndicBERT embeddings are multilingual (includes Indian English sources).

\section{Related Work}

Text Corpora. Few organized sources of monolingual corpora exist for most Indian languages. The EMILLE/CIIL corpus (McEnery et al., 2000) was an early effort to build corpora for South Asian languages, spanning 14 languages with a total of 92 million words. Wikipedia for Indian languages is small (the largest one, Hindi, has just 40 million words). The Leipzig corpus (Goldhahn et al., 2012) contains small collections of upto 1 million sentences for news and web crawls (average 300K sentences). In addition, there are some language specific corpora for Hindi and Urdu (Bojar et al., 2014; Jawaid et al., 2014). In particular, the HindMonoCorp (Bojar et al., 2014) is one of the few larger Indian language collections (787M tokens).

The CommonCrawl ${ }^{1}$ project crawls webpages in many languages by sampling various websites. Our analysis of a processed crawl for the years 2013-2016 (Buck et al., 2014) for Indian languages revealed that most Indian languages, with the exception of Hindi, Tamil and Malayalam, have few good sentences ( $\geq 10$ words) - in the order of around 50 million words. The OSCAR project (Ortiz Suarez et al., 2019), a recent processing of CommonCrawl, also contains much less data for most Indian languages than our crawls. The CC-

\footnotetext{
${ }^{1}$ https://commoncrawl.org
} 
Net (Wenzek et al., 2019) and C4 (Raffel et al., 2019) projects also provide tools to process common crawl, but the extracted corpora are not provided and require a large amount of processing power. Our monolingual corpora is about 4 times larger than the corresponding OSCAR corpus and two times larger than the corresponding CC-100 corpus (Conneau et al., 2020).

Word Embeddings. Word embeddings have been trained for many Indian languages using limited corpora. The Polyglot (Al-Rfou et al., 2013) and FastText (Bojanowski et al., 2017) projects provide embeddings trained on Wikipedia. FastText also provides embeddings trained on Wikipedia + CommonCrawl corpora. We show that on most evaluation tasks IndicFT outperforms existing Fast Text based embeddings.

Pretrained Transformers. Pre-trained transformers serve as general language understanding models that can be used in a wide variety of downstream NLP tasks (Radford et al., 2019). Several transformer-based language models such as GPT (Radford, 2018), BERT (Devlin et al., 2019), RoBERTa (Liu et al., 2019), ALBERT (Lan et al., 2020), etc. have been proposed. All these models require large amounts of monolingual corpora for training. For Indic languages, two such multilingual models are available: XLM-R (Conneau et al., 2020) and multilingual BERT (Devlin et al., 2019). However, they are trained across $\sim 100$ languages and smaller Indic language corpora.

NLU Benchmarks. Benchmarks such as GLUE (Wang et al., 2018), SuperGLUE (Wang et al., 2019), CLUE (Chinese) (Xu et al., 2020), and FLUE (French) (Le et al., 2020) are important for tracking the efficacy of NLP models across languages. Such a benchmark is missing for Indic languages and the goal of this work is to fill this void. Datasets are available for some tasks for a few languages. The following are some of the prominent publicly available datasets ${ }^{2}$ : word similarity (Akhtar et al., 2017), word analogy (Grave et al., 2018), text classification, sentiment analysis (Akhtar et al., 2016; Mukku and Mamidi, 2017), paraphrase detection (Anand Kumar et al., 2016), QA (Clark et al., 2020; Gupta et al., 2018), discourse mode classification (Dhanwal et al., 2020), etc. We also create datasets for some tasks, most of which span all major Indian languages. We bun-

\footnotetext{
${ }^{2} \mathrm{~A}$ comprehensive list of resources for Indian language NLP can be found here: https://github.com/AI4Bharat/indicnlp_catalog
}

\begin{tabular}{lllrrr}
\hline \multicolumn{2}{c}{ Language } & \#S & \#T & \#V & I/O \\
\hline Punjabi & (pa) & 29.2 & 773 & 3.0 & 22 \\
Hindi & $(\mathrm{hi})$ & 63.1 & 1,860 & 6.5 & 2 \\
Bengali & (bn) & 39.9 & 836 & 6.6 & 2 \\
Odia & $(\mathrm{or})$ & 6.94 & 107 & 1.4 & 9 \\
Assamese & $(\mathrm{as)}$ & 1.39 & 32.6 & 0.8 & 8 \\
Gujarati & $(\mathrm{gu})$ & 41.1 & 719 & 5.7 & 14 \\
Marathi & $(\mathrm{mr})$ & 34.0 & 551 & 5.8 & 7 \\
\hline Kannada & $(\mathrm{kn})$ & 53.3 & 713 & 11.9 & 14 \\
Telugu & $(\mathrm{te})$ & 47.9 & 674 & 9.4 & 8 \\
Malayalam & $(\mathrm{ml})$ & 50.2 & 721 & 17.7 & 8 \\
Tamil & $(\mathrm{ta})$ & 31.5 & 582 & 11.4 & 2 \\
\hline English & $(\mathrm{en})$ & 54.3 & 1,220 & 4.5 & \\
\hline Total & & 452.8 & 8789 & 84.7 & \\
\hline
\end{tabular}

Table 1: IndicCorp de-duplicated monolingual corpora statistics: number of sentences (S), tokens $(\mathrm{T})$, types (V) in millions, the ratio of IndicCorp size to OSCAR corpus size (I/O).

dle together the existing datasets and our newly created datasets to create the IndicGLUE benchmark.

\section{IndicCorp: Indian Language Corpora}

In this section, we describe the creation of our monolingual corpora.

Data sources. Our goal was the collection of corpora that reflect contemporary use of Indic languages and cover a wide range of topics. Hence, we focus primarily on crawling news articles, magazines and blogposts. We source our data from popular Indian language news websites. We discover most of our sources through online newspaper directories (e.g., w3newspaper) and automated web searches using hand-picked terms in various languages.

We analyzed whether we could augment our crawls with data from other smaller sources like Leipzig corpus (Goldhahn et al., 2012), WMT NewsCrawl, WMT CommonCrawl (Buck et al., 2014), HindEnCorp (Hindi) (Bojar et al., 2014), etc. Amongst these we chose to augment our dataset with only the CommonCrawl data from the OSCAR corpus (Ortiz Suárez et al., 2019).

Article Extraction. For many news websites, we used BoilerPipe ${ }^{3}$, a tool to automatically extract the main article content for structured pages without any site-specific customizations (Kohlschütter et al., 2010). This approach works well for most of the Indian language news websites. In some cases, we wrote custom extractors for each website

\footnotetext{
${ }^{3}$ https://github.com/kohlschutter/boilerpipe
} 
using BeautifulSoup ${ }^{4}$, a Python library for parsing HTML/XML documents. After content extraction, we applied filters on content length, script, etc., to select good quality articles.

Text Processing. First, we canonicalize the representation of Indic language text in order to handle multiple Unicode representations of certain characters. Next, we split the article into sentences and tokenize the sentences. These steps take into account Indic punctuations and sentence delimiters. Heuristics avoid creating sentences for initials (P. G. Wodehouse) and common Indian titles (Shri., equivalent to Mr. in English) which are followed by a period. We use the Indic NLP Library ${ }^{5}$ (Kunchukuttan, 2020) for processing.

The final corpus for a language is created after combining our crawls with OSCAR corpus ${ }^{6}$ and de-duplicating and shuffling sentences. We used the Murmurhash algorithm (mmh3 Python library with a 128-bit unsigned hash) for de-duplication. Due to copyright reasons, we only release the final shuffled corpus described below.

Dataset Statistics. Table 1 shows statistics of the de-duplicated monolingual datasets for each language. Hindi and Indian English are the largest collections, while Odia and Assamese have the smallest collection. All other languages contain between 500-1000 million tokens. OSCAR is an important contributor to our corpus and accounts for nearly $(23 \%)$ of our corpus by the number of sentences. The rest of the data originated from our crawls. As evident from the last column of Table 1, for 8 languages the number of tokens in our corpus is at least 7 times that in OSCAR. For the remaining 3 languages it is twice that of OSCAR.

\section{IndicGLUE: Multilingual NLU Benchmark}

We now introduce Indic GLUE, the Indic General Language Understanding Evaluation Benchmark, which is a collection of various NLP tasks as described below. The goal is to provide an evaluation benchmark for natural language understanding capabilities of NLP models on diverse tasks and multiple Indian languages. As discussed earlier, very few public NLP datasets are available for all Indian languages. Hence, we adopted a two-pronged approach to construct this benchmark. One, we use

\footnotetext{
${ }^{4}$ https://www.crummy.com/software/BeautifulSoup

${ }^{5}$ https://github.com/anoopkunchukuttan/indic_nlp_library

${ }^{6}$ https://oscar-corpus.com/
}

existing datasets that address some tasks. However, such datasets are available for just 4-5 Indian languages. We also manually translated some English datasets into a few Indian languages. We summarize statistics of these datasets in Appendix A. Two, we create new datasets that span all major Indian languages. These datasets are curated semi-automatically using external metadata like website/Wikipedia structure and are designed to present reasonably complex NLU tasks. Table 2 summarizes the sizes of the respective datasets. Further details (such as the min, max, average number of words per training instance) can be found in Appendix C. Standard train and test splits for all datasets are publicly available on the website for reproducibility. For publicly available datasets, we used the original split if provided.

News Category Classification. The task is to predict the genre/topic of a given news article or news headline. We create news article category datasets using IndicCorp for 9 languages. The categories are determined from URL components. We chose generic categories which are likely to be consistent across websites (e.g., entertainment, sports, business, lifestyle, technology, politics, crime) . See Appendix B for details.

Headline Prediction Task. The task is to predict the correct headline for a news article from a given list of four candidate headlines ( 3 incorrect, 1 correct). We generate the dataset from our news article crawls which contain articles and their headlines. We ensure that the three incorrect candidates are not completely unrelated to the given article. In particular, while choosing incorrect candidates, we considered only those articles that had a sizeable overlap of entities with the original article.

Wikipedia Section-title Prediction. The task is to predict the correct title for a Wikipedia section from a given list of four candidate titles (3 incorrect, 1 correct). We use the open-source tool WikiExtractor to extract sections and their titles from Wikipedia. To make the task challenging, we choose the 3 incorrect candidates for a given section, only from the titles of other sections in the same article as the given section.

Cloze-style Multiple-choice QA. Given a text with an entity randomly masked, the task is to predict that masked entity from a list of 4 candidate entities (3 incorrect, 1 correct). The text is obtained from Wikipedia articles and the entities in the text are identified using Wikidata. We choose the 3 in- 


\begin{tabular}{|c|c|c|c|c|c|c|c|c|c|c|c|}
\hline pa & hi & bn & or & as & $\mathrm{gu}$ & $\mathrm{mr}$ & $\mathrm{kn}$ & te & $\mathrm{ml}$ & ta & total \\
\hline \multicolumn{12}{|c|}{ News Category Classification } \\
\hline 3,120 & - & 14,000 & 30,000 & - & 2,040 & 4,770 & 30,000 & 24,000 & 6,000 & 11,700 & 125,630 \\
\hline \multicolumn{12}{|c|}{ Headline Prediction } \\
\hline 100,000 & 100,000 & 68,350 & 100,000 & 49,751 & 100,000 & 67,571 & 56,457 & 63,415 & 100,000 & 74,767 & 880,311 \\
\hline \multicolumn{12}{|c|}{ Wikipedia Section-Title Prediction } \\
\hline 10,966 & 55,087 & 59,475 & 5,019 & 6,251 & 12,506 & 13,058 & 44,224 & 100,000 & 34,409 & 61,175 & 402,170 \\
\hline \multicolumn{12}{|c|}{ Cloze-style QA } \\
\hline 5,664 & 35,135 & 38,845 & 1,975 & 2,942 & 22,856 & 11,370 & 13,656 & 41,338 & 26,531 & 38,585 & 238,897 \\
\hline \multicolumn{12}{|c|}{ Named Entity Recognition } \\
\hline 9,462 & 69,431 & 109,508 & 8,687 & 6,295 & 39,708 & 108,579 & 28,854 & 81,627 & 138,888 & 186,423 & 787,462 \\
\hline \multicolumn{12}{|c|}{ Cross-lingual Sentence Retrieval (\#English to Indian language parallel sentences) } \\
\hline- & 5,169 & 5,522 & 752 & - & 6,463 & 5,760 & - & 5,049 & 4,886 & 5,637 & 39,238 \\
\hline
\end{tabular}

Table 2: IndicGLUE Datasets' Statistics. The first four datasets have been created as part of this project.

correct candidates from entities that occur in the same article and have the same type as the correct entity. The type of an entity is taken from Wikidata. This task is similar to the one proposed by Petroni et al. (2019) for English, and aims to check if language models can be used as knowledge bases.

Named Entity Recognition. We use the WikiAnn NER dataset ${ }^{7}$ (Pan et al., 2017) which contains NER data for 282 languages. This dataset is created from Wikipedia by utilizing cross language links to propagate English named entity labels to other languages. We consider the following coarsegrained labels in this dataset: Person (PER), Organisation (ORG) and Location (LOC).

Cross-lingual Sentence Retrieval. Given a sentence in English, the task is to retrieve its translation from a set of candidate sentences in an Indian language. We use the CVIT-Mann Ki Baat dataset ${ }^{8}$ (Siripragrada et al., 2020) for this task.

Winograd NLI (WNLI). The WNLI task (Levesque et al., 2011) is part of the GLUE benchmark. Each example in the dataset consists of a pair of sentences where the second sentence is constructed from the first sentence by replacing an ambiguous pronoun with a possible referent within the sentence. The task is to predict if the second sentence is entailed by the original sentence. We manually translated this dataset to 3 Indic languages (hi, $\mathrm{mr}, \mathrm{gu}$ ) with the help of skilled bilingual speakers. The annotators were paid 3 cents per word and the translations were then verified by an expert bilingual speaker.

\footnotetext{
${ }^{7}$ https://elisa-ie.github.io/wikiann/

${ }^{8} \mathrm{http}: / /$ preon.iiit.ac.in/ jerin/bhasha/
}

COPA. The Choice Of Plausible Alternatives (Gordon et al., 2011) task evaluates open-domain commonsense causal reasoning. It consists of a large set of 2-choice questions, formulated as a premise and two alternatives written as sentences. The task is to select the alternative that is more plausibly the cause (or effect) of the situation described by the premise. As with WNLI, we translated the dataset into 3 Indic languages (hi, mr, gu).

Paraphrase Detection. We use the Amritha paraphrase dataset comprsing 4 Indic languages (hi,pa,ta,ml) (Anand Kumar et al., 2016). We evaluate on two subtasks: Subtask 1- Given a pair of sentences from news paper domain, the task is to classify them as paraphrases $(\mathrm{P})$ or not paraphrases (NP). Subtask 2- Given two sentences from news paper domain, the task is to identify whether they are completely equivalent (E) or roughly equivalent (RE) or not equivalent (NE). This task is similar to subtask 1, but the main difference is the use of three classes instead of two.

Discourse Mode Classification. Given a sentence, the task is to classify it into one of the following discourse categories: argumentative, descriptive, dialogic, informative, narrative. We use the MIDAS Hindi Discourse Analysis dataset (Dhanwal et al., 2020) for this task.

Sentiment Analysis. We used the following publicly available datasets: (a) IIT-Patna Movie and Product Sentiment Analysis dataset (Hindi) (Akhtar et al., 2016), (b) ACTSA Sentiment Analysis corpus (Telugu) (Mukku and Mamidi, 2017). 


\begin{tabular}{|c|c|c|c|}
\hline Lang & FT-W & FT-WC & IndicFT \\
\hline \multicolumn{4}{|c|}{ Word Similarity (Pearson Correlation) } \\
\hline pa & 0.467 & 0.384 & 0.445 \\
\hline hi & 0.575 & 0.551 & 0.598 \\
\hline $\mathrm{gu}$ & 0.507 & 0.521 & 0.600 \\
\hline $\mathrm{mr}$ & 0.497 & 0.544 & 0.509 \\
\hline te & 0.559 & 0.543 & 0.578 \\
\hline ta & 0.439 & 0.438 & 0.422 \\
\hline Average & 0.507 & 0.497 & 0.525 \\
\hline \multicolumn{4}{|c|}{ Word Analogy (\% accuracy) } \\
\hline hi & 19.76 & 32.93 & 29.65 \\
\hline
\end{tabular}

Table 3: Word Similarity and Analogy Results for different pre-trained embeddings. (a) FT-W: FastText Wikipedia, (b) FT-WC: FastText Wikipedia + CommonCrawl, (c) IndicFT: IndicNLP.

\section{IndicFT: Word Embeddings}

We train FastText word embeddings for each language using IndicCorp, and evaluate their quality on: (a) word similarity, (b) word analogy, (c) text classification, (d) bilingual lexicon induction tasks. We compare our embeddings (referred to as IndicFT) with two pre-trained embeddings released by the FastText project trained on Wikipedia (FT-W) (Bojanowski et al., 2017) and Wiki+CommonCrawl (FT-WC) (Grave et al., 2018) respectively.

\subsection{Training Details}

We train 300-dimensional word embeddings for each language on IndicCorp using FastText (Bojanowski et al., 2017). Since Indian languages are morphologically rich, we chose FastText, which is capable of integrating subword information by using character $n$-gram embeddings during training. We train skipgram models for 10 epochs with a window size of 5 , minimum token count of 5 and 10 negative examples sampled for each instance. We chose these hyper-parameters based on suggestions by Grave et al. (2018). Based on previously published results, we expect FastText to be better than word-level algorithms like word2vec (Mikolov et al., 2013b) and GloVe (Pennington et al., 2014) for morphologically rich languages.

\subsection{Word Similarity \& Analogy Evaluation}

We perform an intrinsic evaluation of the word embeddings using the IIIT-Hyderabad word similarity dataset (Akhtar et al., 2017) (7 Indian languages with 100-200 word-pairs per language) and the Facebook Hindi word analogy dataset (Grave et al., 2018). Table 3 shows the evaluation results.

\begin{tabular}{llrrr}
\hline Lang & Dataset & FT-W & FT-WC & IndicFT \\
\hline hi & BBC Articles & 72.29 & 67.44 & $\mathbf{7 7 . 0 2}$ \\
& IITP+ Movie & 41.61 & 44.52 & $\mathbf{4 5 . 8 1}$ \\
& IITP Product & 58.32 & 57.17 & $\mathbf{6 1 . 5 7}$ \\
\hline bn & Soham Articles & 62.79 & 64.78 & $\mathbf{7 1 . 8 2}$ \\
\hline gu & & 81.94 & 84.07 & $\mathbf{9 0 . 7 4}$ \\
ml & iNLTK & 86.35 & 83.65 & $\mathbf{9 5 . 8 7}$ \\
mr & Headlines & 83.06 & 81.65 & $\mathbf{9 1 . 4 0}$ \\
ta & & 90.88 & 89.09 & $\mathbf{9 5 . 3 7}$ \\
\hline te & ACTSA & 46.03 & 42.51 & $\mathbf{5 2 . 5 8}$ \\
\hline & Average & 69.25 & 68.32 & $\mathbf{7 5 . 8 0}$ \\
\hline
\end{tabular}

Table 4: Text classification accuracy on public datasets.

On average, IndicFT embeddings outperform the baseline embeddings.

\subsection{Text Classification Evaluation}

We evaluated the embeddings on different text classification tasks: (a) news article topic, (b) news headlines topic and (c) sentiment classification.

Datasets. In addition to the IndicGLUE News Category dataset, we experimented on the following publicly available datasets: (a) IIT-Patna Sentiment Analysis dataset (Akhtar et al., 2016), (b) ACTSA Sentiment Analysis corpus (Mukku and Mamidi, 2017), (c) BBC News Articles classification dataset, (d) iNLTK Headlines dataset, and (e) Soham Bengali News classification dataset. (See Appendix A for dataset details).

Classifier training. Following Meng et al. (2019), we use a $k$-NN $(k=4)$ classifier since it is nonparameteric. Hence, classification performance directly reflects how well the embedding space captures text semantics. The input text embedding is the mean of all word embeddings.

Results. On nearly all datasets and languages, IndicFT embeddings outperform baseline embeddings (see Tables 4 and 5).

\subsection{Bilingual Lexicon Induction}

We train bilingual word embeddings from English to Indian languages and vice versa using GeoMM (Jawanpuria et al., 2019), a state-of-the-art supervised method for learning bilingual embeddings. We evaluate the bilingual embeddings on the BLI task, using bilingual dictionaries from the MUSE project and a en-te dictionary created in-house. We search among the 200k most frequent target language words with the CSLS distance metric during inference (Conneau et al., 2018). Table 6 shows the results. The quality of multilingual embed- 


\begin{tabular}{lrrr}
\hline \multicolumn{1}{c}{ Lang } & FT-W & FT-WC & IndicFT \\
\hline pa & $\mathbf{9 7 . 1 2}$ & 95.53 & 96.47 \\
bn & 96.57 & 97.57 & $\mathbf{9 7 . 7 1}$ \\
or & 94.80 & 96.20 & $\mathbf{9 8 . 4 3}$ \\
gu & 95.12 & 94.63 & $\mathbf{9 9 . 0 2}$ \\
mr & 96.44 & 97.07 & $\mathbf{9 9 . 3 7}$ \\
\hline kn & 95.93 & 96.53 & $\mathbf{9 7 . 4 3}$ \\
te & 98.67 & 98.08 & $\mathbf{9 9 . 1 7}$ \\
ml & 89.02 & 89.18 & $\mathbf{9 2 . 8 3}$ \\
ta & 95.99 & 95.90 & $\mathbf{9 7 . 2 6}$ \\
\hline Average & 95.52 & 95.63 & $\mathbf{9 7 . 5 2}$ \\
\hline
\end{tabular}

Table 5: Accuracy on IndicGLUE News category testset.

\begin{tabular}{llllllll}
\hline & \multicolumn{3}{c}{ en to Indic } & & \multicolumn{3}{c}{ Indic to en } \\
\cline { 2 - 3 } \cline { 7 - 8 } & FT-W & FT-WC & Ours & & FT-W & FT-WC & Ours \\
\hline bn & 22.60 & 33.92 & $\mathbf{3 6 . 6 8}$ & & 31.22 & 42.10 & $\mathbf{4 2 . 6 7}$ \\
hi & 40.93 & $\mathbf{4 4 . 3 5}$ & 41.53 & & 49.56 & $\mathbf{5 7 . 1 6}$ & 54.85 \\
te & 21.10 & 23.01 & $\mathbf{5 1 . 1 1}$ & & 25.36 & 32.84 & $\mathbf{5 7 . 5 8}$ \\
ta & 19.27 & 30.25 & $\mathbf{3 1 . 8 7}$ & & 26.66 & $\mathbf{4 0 . 2 0}$ & 38.65 \\
\hline Ave. & 25.98 & 32.88 & $\mathbf{4 0 . 2 9}$ & & 33.20 & 43.08 & $\mathbf{4 8 . 3 8}$ \\
\hline
\end{tabular}

Table 6: Accuracy@1 for BLI. Ours refers to IndicFT.

dings depends on the quality of monolingual embeddings. IndicFT bilingual embeddings significantly outperform the baseline bilingual embeddings for most languages.

\section{IndicBERT: Multilingual NLU Model}

In this section, we introduce IndicBERT which is trained on Indic Corp and evaluated on Indic GLUE. We specifically chose ALBERT as the base model as it has fewer parameters making it easier to distribute and use in downstream applications. Further, similar to mBERT, we chose to train a single model for all Indian languages with a hope of utilizing the relatedness amongst Indian languages. In particular, such joint training may be beneficial for some of the under-represented languages (e.g., Odia and Assamese).

\subsection{Pre-training}

Using IndicCorp we first train a sentence piece tokenizer (Kudo and Richardson, 2018) to tokenize the sentences in each language. We use this tokenized corpora to train a multilingual ALBERT using the standard masked language model (MLM) objective. Note that we did not use the Sentence Order Prediction objective used in the original ALBERT work. Similar to mBERT and XLM-R models, we perform exponentially smoothed weighting of the data across languages to give a better representation to low-resource languages. We choose a vocabulary of $200 \mathrm{k}$ to accommodate different scripts and large vocabularies of Indic languages.

We train our models on a single TPU v3 provided by Tensorflow Research Cloud ${ }^{9}$. We train both the base and large versions of ALBERT. To account for memory constraints, we use a smaller maximum sequence length of 128 . In addition, for the large model, we use a smaller batch size of 2048. For creating each batch, we first randomly select a language and then randomly select sentences from that language. Apart from sequence length and batch size, we use the default values for the remaining hyperparameters as in Lan et al. (2020). We train the model for a total of 400k steps. It took 6 days to train the base model and 9 days to train the large model. In the remaining discussion, we refer to our models as IndicBERT base and IndicBERT large. Our models are compared with two of the best performing multilingual models: mBERT (Pires et al., 2019) and XLM-R base model (Ruder et al., 2019). Not that our model is much smaller compared to these models, while it is trained on larger Indic language corpora (see Table 14 in Appendix C.5 for details).

\subsection{Fine-tuning}

After pre-training, we fine-tune IndicBERT on each of the tasks in IndicGLUE using the respective training sets. The fine-tuning is done independently for each task and each language (i.e., we have a task-specific model for each language). We describe the fine-tuning procedure for each task.

Headline Prediction, Wikipedia Section Title Prediction. For headline prediction, we feed the article and candidate headline to the model with a SEP token in between. We have a classification head at the top which assigns a score between 0 and 1 to the headline. We use cross entropy loss with the target label as 1 for the correct candidate and 0 for the incorrect candidates. During prediction, we choose the candidate headline assigned the highest score. Section title prediction uses the same procedure (Wikipedia section and section titles instead of news articles and headlines respectively).

Named Entity Recognition. Each sentence is fed as a single sequence to the model. For every token, we have a softmax layer at the output which computes a probability distribution over the NER

\footnotetext{
${ }^{9} \mathrm{https}: / /$ www.tensorflow.org/tfrc
} 


\begin{tabular}{lcccccccccccc}
\hline Model & pa & hi & bn & or & as & gu & mr & kn & te & ml & ta & avg \\
\hline News Article Headline Prediction & & & & & & & & & & \\
XLM-R & 97.44 & 94.72 & 94.62 & 93.20 & 96.14 & 97.28 & $\mathbf{9 4 . 7 9}$ & 98.16 & 91.30 & 96.32 & 96.90 & 95.52 \\
mBERT & 94.32 & 94.56 & 90.64 & 52.64 & 92.92 & 94.24 & 90.77 & 96.88 & 88.40 & 94.24 & 95.72 & 89.58 \\
IndicBERT base & 97.36 & 95.36 & $\mathbf{9 5 . 9 1}$ & $\mathbf{9 3 . 8 4}$ & 96.62 & 97.36 & 93.85 & 97.88 & 89.16 & $\mathbf{9 6 . 4 8}$ & 96.26 & 95.46 \\
IndicBERT large & $\mathbf{9 7 . 6 8}$ & $\mathbf{9 5 . 6 8}$ & 95.79 & 93.28 & $\mathbf{9 7 . 4 3}$ & $\mathbf{9 7 . 9 2}$ & 93.14 & $\mathbf{9 8 . 1 6}$ & $\mathbf{9 2 . 6 9}$ & 95.20 & $\mathbf{9 7 . 6 5}$ & $\mathbf{9 5 . 8 7}$ \\
\hline Wikipedia Section Title Prediction & & & & & & & & & & & \\
XLM-R & 70.29 & 76.92 & 80.91 & 68.25 & 56.96 & 27.39 & 77.44 & 24.41 & 94.64 & $\mathbf{7 6 . 1 0}$ & 76.34 & 66.33 \\
mBERT & 72.47 & $\mathbf{8 0 . 1 2}$ & 82.53 & 22.22 & $\mathbf{7 3 . 4 2}$ & $\mathbf{7 4 . 5 2}$ & $\mathbf{8 0 . 4 9}$ & 78.84 & 94.56 & 74.25 & 76.86 & $\mathbf{7 3 . 6 6}$ \\
IndicBERT base & 67.39 & 74.02 & 80.11 & 57.14 & 65.82 & 68.79 & 72.56 & 75.05 & 94.80 & 75.87 & 74.90 & 73.31 \\
IndicBERT large & $\mathbf{7 7 . 5 4}$ & 77.80 & $\mathbf{8 2 . 6 6}$ & $\mathbf{6 8 . 2 5}$ & 56.96 & 52.23 & 77.44 & $\mathbf{8 0 . 1 1}$ & $\mathbf{9 5 . 3 6}$ & 64.27 & 71.37 & 73.09 \\
\hline Cloze-style multiple-choice & $\mathbf{Q A}$ & & & & & & & & & & \\
XLM-R & 29.31 & 30.62 & 29.95 & 35.98 & 27.11 & 11.15 & 32.38 & 29.36 & 27.16 & 27.57 & 27.24 & 27.98 \\
mBERT & 33.70 & 39.00 & 36.23 & 26.37 & 29.42 & $\mathbf{8 3 . 3 1}$ & 38.81 & 33.96 & $\mathbf{3 7 . 5 8}$ & $\mathbf{3 6 . 7 1}$ & $\mathbf{3 5 . 7 2}$ & 39.16 \\
IndicBERT base & $\mathbf{4 4 . 7 4}$ & $\mathbf{4 1 . 5 5}$ & $\mathbf{3 9 . 4 0}$ & $\mathbf{3 9 . 3 2}$ & $\mathbf{4 0 . 4 9}$ & 70.78 & $\mathbf{4 4 . 8 5}$ & $\mathbf{3 9 . 5 7}$ & 32.60 & 35.39 & 31.83 & $\mathbf{4 1 . 8 7}$ \\
IndicBERT large & 41.91 & 37.01 & 32.63 & 33.81 & 30.03 & 52.73 & 39.98 & 32.28 & 26.73 & 28.04 & 28.10 & 34.84 \\
\hline
\end{tabular}

Table 7: Test accuracy on various multiple-choice tasks.

\begin{tabular}{lcccccccccccc}
\hline Model & $\mathrm{pa}$ & $\mathrm{hi}$ & $\mathrm{bn}$ & $\mathrm{or}$ & $\mathrm{as}$ & $\mathrm{gu}$ & $\mathrm{mr}$ & $\mathrm{kn}$ & $\mathrm{te}$ & $\mathrm{ml}$ & ta & avg \\
\hline Article Genre Classification & & & & & & & & & & \\
XLM-R & 94.87 & - & $\mathbf{9 8 . 2 9}$ & 97.07 & - & 96.15 & 96.67 & 97.60 & 99.33 & $\mathbf{9 6 . 0 0}$ & $\mathbf{9 7 . 2 8}$ & 97.03 \\
mBERT & 94.87 & - & 97.71 & 69.33 & - & 84.62 & 96.67 & 97.87 & 98.67 & 81.33 & 94.56 & 90.63 \\
IndicBERT base & $\mathbf{9 7 . 4 4}$ & - & 97.14 & 97.33 & - & $\mathbf{1 0 0 . 0 0}$ & $\mathbf{9 6 . 6 7}$ & 97.87 & 99.67 & 93.33 & 96.60 & $\mathbf{9 7 . 3 4}$ \\
IndicBERT large & 94.87 & - & 97.71 & $\mathbf{9 7 . 6 0}$ & - & 73.08 & 95.00 & $\mathbf{9 7 . 8 7}$ & $\mathbf{9 9 . 6 7}$ & 85.33 & 95.24 & 92.93 \\
\hline Named Entity Recognition (F1-score) & & & & & & & & & & & & \\
XLM-R & 17.86 & 89.62 & $\mathbf{9 2 . 9 5}$ & 25.00 & 66.67 & 55.32 & 87.86 & 47.06 & 81.71 & 81.98 & 79.16 & 65.93 \\
mBERT & $\mathbf{5 0 . 0 0}$ & 86.56 & 91.81 & 19.05 & $\mathbf{9 2 . 3 1}$ & 68.04 & $\mathbf{9 1 . 2 7}$ & 59.72 & 84.31 & 82.64 & 79.90 & $\mathbf{7 3 . 2 4}$ \\
IndicBERT base & 21.43 & $\mathbf{9 0 . 3 0}$ & 93.39 & 8.69 & 41.67 & 54.74 & 88.71 & 52.29 & $\mathbf{8 4 . 3 8}$ & 83.16 & $\mathbf{9 0 . 4 5}$ & 64.47 \\
IndicBERT large & 44.44 & 86.81 & 91.85 & $\mathbf{3 5 . 0 9}$ & 43.48 & $\mathbf{7 0 . 2 1}$ & 87.73 & $\mathbf{6 3 . 5 1}$ & 80.12 & $\mathbf{8 4 . 3 5}$ & 80.81 & 69.85 \\
\hline
\end{tabular}

Table 8: Test accuracy on various classification tasks.

classes. We fine-tune the model using multi-class cross entropy loss.

Cloze-style Multiple-choice QA. We feed the masked text segment as input to the model and at the output we have a softmax layer which predicts a probability distribution over the given candidates. We fine-tune the model using cross entropy loss with the target label as 1 for the correct candidate and 0 for the incorrect candidates.

Cross-lingual Sentence Retrieval. No finetuning is required for this task. We compute the representation of every sentence by mean-pooling the outputs in the last hidden layer and then using cosine distance to compute similarity between sentences (Libovický et al., 2019). Additionally, we also center the sentence vectors across each language to remove language-specific bias in the vectors (Reimers and Gurevych, 2019).

Winograd NLI, COPA, Paraphrase Detection:
We input the sentence pair into the model as segment A and segment $\mathrm{B}$. The [CLS] representation from the last layer is fed into an output layer for classification into one of the categories.

News Category Classification, Discourse Mode Classification, Sentiment Analysis. We feed the representation of the [CLS] token from the last layer to a linear classifier with a softmax layer to predict a probability distribution over the categories. We fine-tune the model using multi-class cross entropy loss.

\subsection{Evaluation}

We summarize the main observations from our results as reported in Tables 7-10.

Comparison with mBERT and XLM-R. On most tasks, IndicBERT models outperform XLM$\mathrm{R}$ and mBERT. Specifically, IndicBERT models are competitive on the Wikipedia Section Title pre- 


\begin{tabular}{|c|c|c|c|c|}
\hline Language & Dataset & Ours & mBERT & XLM-R \\
\hline \multicolumn{5}{|c|}{ Article Genre Classification } \\
\hline hi & BBC News & 74.60 & 60.55 & 75.52 \\
\hline bn & Soham Articles & 78.45 & 80.23 & 87.60 \\
\hline gu & INLTK Headlines & 92.91 & 89.16 & 89.83 \\
\hline $\mathrm{ml}$ & INLTK Headlines & 94.76 & 82.28 & 95.40 \\
\hline $\mathrm{mr}$ & INLTK Headlines & 94.30 & 87.50 & 92.48 \\
\hline ta & INLTK Headlines & 96.11 & 92.86 & 95.81 \\
\hline \multicolumn{5}{|c|}{ Sentiment Analysis } \\
\hline hi & Product Reviews & 71.32 & 74.57 & 78.97 \\
\hline hi & Movie Reviews & 59.03 & 56.77 & 61.61 \\
\hline te & ACTSA & 61.18 & 48.53 & 59.33 \\
\hline \multicolumn{5}{|c|}{ Discourse Mode Classification } \\
\hline hi & MIDAS Discourse & 78.44 & 71.20 & 79.94 \\
\hline \multicolumn{5}{|c|}{ Semantic Similarity } \\
\hline hi & Amrita Subtask 1 & 93.11 & 93.22 & 91.78 \\
\hline ta & Amrita Subtask 1 & 92.78 & 93.33 & 92.11 \\
\hline $\mathrm{ml}$ & Amrita Subtask 1 & 89.11 & 88.67 & 88.78 \\
\hline pa & Amrita Subtask 1 & 100.00 & 100.00 & 99.40 \\
\hline hi & Amrita Subtask 2 & 85.79 & 87.29 & 88.20 \\
\hline ta & Amrita Subtask 2 & 69.07 & 68.57 & 68.21 \\
\hline $\mathrm{ml}$ & Amrita Subtask 2 & 89.00 & 84.44 & 84.67 \\
\hline pa & Amrita Subtask 2 & 93.47 & 93.20 & 87.73 \\
\hline \multicolumn{5}{|c|}{ Textual Entailment } \\
\hline hi & WNLI & 56.34 & 56.34 & 54.93 \\
\hline $\mathrm{mr}$ & WNLI & 56.34 & 56.34 & 56.34 \\
\hline gu & WNLI & 56.34 & 56.34 & 56.34 \\
\hline hi & COPA & 62.50 & 65.91 & 43.18 \\
\hline $\mathrm{mr}$ & COPA & 59.09 & 55.68 & 61.36 \\
\hline \multirow[t]{2}{*}{ gu } & COPA & 53.41 & 43.18 & 48.86 \\
\hline & Average & 77.39 & 74.42 & 76.60 \\
\hline
\end{tabular}

Table 9: Test Accuracies on public datasets. Ours refer to IndicBERT-base.

diction task, but are out-performed by mBERT on the NER dataset. On the publicly available datasets (Table 9), IndicBERT-base outpeforms the existing models.

Performance on Wikipedia Tasks. We notice that the performance of mBERT is relatively higher for the tasks based on Wikipedia data, namely NER, Wikipedia Section Title prediction, and Multiple-choice QA. This suggests that mBERT, unlike other models, is benefiting from exposure to Wikipedia data during pretraining. Note that we deliberately did not include Wikipedia in our monolingual corpora as it is a good source for creating NLU tasks. Hence, we wanted to avoid overlap between our pretraining data and any potential Wikipedia-based dataset.

Small v/s Large IndicBERT. The large and base

\begin{tabular}{lrrrr}
\hline Language & XLM-R & mBERT & IB base & IB large \\
\hline en-hi & 4.77 & $\mathbf{3 3 . 7 3}$ & 24.67 & 21.99 \\
en-bn & 9.46 & 26.30 & 26.12 & $\mathbf{2 9 . 0 0}$ \\
en-or & 15.96 & 2.66 & 33.11 & $\mathbf{4 9 . 6 0}$ \\
en-gu & 18.46 & 17.68 & 28.17 & $\mathbf{3 9 . 4 3}$ \\
en-mr & 18.07 & 24.67 & 23.09 & $\mathbf{3 2 . 6 7}$ \\
en-te & 15.23 & 26.13 & 25.10 & $\mathbf{3 4 . 3 0}$ \\
en-ml & 17.47 & 16.76 & 31.22 & $\mathbf{3 2 . 2 6}$ \\
en-ta & 10.48 & 23.78 & 25.44 & $\mathbf{3 3 . 5 8}$ \\
\hline avg & 13.74 & 21.46 & 27.12 & $\mathbf{3 4 . 1 0}$ \\
\hline
\end{tabular}

Table 10: Precision@10 on Cross-Lingual Sentence Retrieval Task.

models of IndicBERT are comparable. There are some tasks on which either task is clearly better.

Challenging tasks. Multiple-choice QA and Cross-Lingual Sentence Retrieval prove to be the more challenging tasks. On both tasks, IndicBERT models improve on XLM-R and mBERT.

Effect of corpus size. Comparing across languages, on the 5 mono-lingual tasks, the performance of IndicBERT large is poorest on Assamese and Odia - the two languages with the smallest corpora sizes. On the other hand, performance is highest on Hindi and Bengali, which have the largest corpora sizes. This reinforces the expectation that accuracy is sensitive to the corpora size.

\section{Conclusion and Future Work}

We present the IndicNLPSuite, a collection of large-scale, general-domain, sentence-level corpora of 8.9 billion words across 11 Indian languages, along with pre-trained models (IndicFT, IndicBERT) and NLU benchmarks (IndicGLUE). We show that resources derived from this dataset outperform other pre-trained embeddings on many NLP tasks. The sentence-level corpora, embeddings and evaluation datasets are publicly available under a Creative Commons AttributionNonCommercial-ShareAlike 4.0 International License. We hope the availability of these resources will accelerate NLP research for Indian languages by enabling the community to build further resources and solutions for various NLP tasks and opening up interesting NLP questions.

\section{Acknowledgments}

We thank Google for providing TPU hardware via the Tensorflow Research Cloud (TFRC) grant. 


\section{References}

Md. Shad Akhtar, Ayush Kumar, Asif Ekbal, and Pushpak Bhattacharyya. 2016. A hybrid deep learning architecture for sentiment analysis. In COLING 2016, 26th International Conference on Computational Linguistics, Proceedings of the Conference: Technical Papers, December 11-16, 2016, Osaka, Japan, pages 482-493.

Syed Sarfaraz Akhtar, Arihant Gupta, Avijit Vajpayee, Arjit Srivastava, and Manish Shrivastava. 2017. Word similarity datasets for Indian languages: Annotation and baseline systems. In Proceedings of the 11th Linguistic Annotation Workshop, pages 91-94, Valencia, Spain. Association for Computational Linguistics.

Rami Al-Rfou, Bryan Perozzi, and Steven Skiena. 2013. Polyglot: Distributed word representations for multilingual nlp. In Proceedings of the Seventeenth Conference on Computational Natural Language Learning, pages 183-192, Sofia, Bulgaria. Association for Computational Linguistics.

M. Anand Kumar, S. Singh, B. Kavirajan, and K.P. Soman. 2016. DPIL@FIRE 2016: Overview of shared task on detecting paraphrases in Indian Languages (DPIL). In CEUR Workshop Proceedings.

Piotr Bojanowski, Edouard Grave, Armand Joulin, and Tomas Mikolov. 2017. Enriching word vectors with subword information. Transactions of the Association for Computational Linguistics, 5:135-146.

Ondrej Bojar, Vojtech Diatka, Pavel Rychlỳ, Pavel Stranák, Vít Suchomel, Ales Tamchyna, and Daniel Zeman. 2014. Hindencorp-hindi-english and hindionly corpus for machine translation. In $L R E C$, pages 3550-3555.

Christian Buck, Kenneth Heafield, and Bas van Ooyen. 2014. N-gram counts and language models from the common crawl. In Proceedings of the Ninth International Conference on Language Resources and Evaluation (LREC'14), pages 3579-3584, Reykjavik, Iceland. European Language Resources Association (ELRA).

Jonathan H. Clark, Eunsol Choi, Michael Collins, Dan Garrette, Tom Kwiatkowski, Vitaly Nikolaev, and Jennimaria Palomaki. 2020. Tydi qa: A benchmark for information-seeking question answering in typologically diverse languages. Transactions of the Association for Computational Linguistics.

Alexis Conneau, Kartikay Khandelwal, Naman Goyal, Vishrav Chaudhary, Guillaume Wenzek, Francisco Guzmán, E. Grave, Myle Ott, L. Zettlemoyer, and V. Stoyanov. 2020. Unsupervised cross-lingual representation learning at scale. In $A C L$.

Alexis Conneau, Guillaume Lample, Marc'Aurelio Ranzato, Ludovic Denoyer, and Hervé Jégou. 2018. Word translation without parallel data. In Proceedings of the International Conference on Learning Representations.
Jacob Devlin, Ming-Wei Chang, Kenton Lee, and Kristina Toutanova. 2019. BERT: Pre-training of deep bidirectional transformers for language understanding. In Proceedings of the 2019 Conference of the North American Chapter of the Association for Computational Linguistics: Human Language Technologies, Volume 1 (Long and Short Papers), pages 4171-4186, Minneapolis, Minnesota. Association for Computational Linguistics.

Swapnil Dhanwal, Hritwik Dutta, Hitesh Nankani, Nilay Shrivastava, Yaman Kumar, Junyi Jessy Li, Debanjan Mahata, Rakesh Gosangi, Haimin Zhang, Rajiv Ratn Shah, and Amanda Stent. 2020. An annotated dataset of discourse modes in Hindi stories. In Proceedings of The 12th Language Resources and Evaluation Conference, pages 1191-1196, Marseille, France. European Language Resources Association.

Murray B Emeneau. 1956. India as a lingustic area. Language.

D. Goldhahn, T. Eckart, and U. Quasthoff. 2012. Building large monolingual dictionaries at the leipzig corpora collection: From 100 to 200 languages. In Proceedings of the International Conference on Language Resources and Evaluation (LREC 2012).

Andrew S. Gordon, Zornitsa Kozareva, and Melissa Roemmele. 2011. Semeval-2012 task 7: Choice of plausible alternatives: An evaluation of commonsense causal reasoning. In SemEval@NAACL-HLT.

Edouard Grave, Piotr Bojanowski, Prakhar Gupta, Armand Joulin, and Tomas Mikolov. 2018. Learning word vectors for 157 languages. In Proceedings of the International Conference on Language Resources and Evaluation (LREC 2018).

Deepak Gupta, Surabhi Kumari, Asif Ekbal, and Pushpak Bhattacharyya. 2018. MMQA: A Multi-domain Multi-lingual Question-Answering Framework for English and Hindi. In Proceedings of the Eleventh International Conference on Language Resources and Evaluation (LREC 2018), Miyazaki, Japan. European Language Resources Association (ELRA).

Bushra Jawaid, Amir Kamran, and Ondrej Bojar. 2014. A Tagged Corpus and a Tagger for Urdu. In $L R E C$, pages 2938-2943.

Pratik Jawanpuria, Arjun Balgovind, Anoop Kunchukuttan, and Bamdev Mishra. 2019. Learning multilingual word embeddings in latent metric space: a geometric approach. Transaction of the Association for Computational Linguistics (TACL), 7:107-120.

Christian Kohlschütter, Péter Fankhauser, and Wolfgang Nejdl. 2010. Boilerplate detection using shallow text features. In WSDM.

Taku Kudo and John Richardson. 2018. SentencePiece: A simple and language independent subword tokenizer and detokenizer for neural text processing. In Proceedings of the 2018 Conference on Empirical 
Methods in Natural Language Processing: System Demonstrations, pages 66-71, Brussels, Belgium. Association for Computational Linguistics.

Anoop Kunchukuttan. 2020. The IndicNLP Library. https://github.com/anoopkunchukuttan/indic_ nlp_library/blob/master/docs/indicnlp.pdf.

Zhenzhong Lan, Mingda Chen, Sebastian Goodman, Kevin Gimpel, Piyush Sharma, and Radu Soricut. 2020. Albert: A lite bert for self-supervised learning of language representations. ArXiv, abs/1909.11942.

Hang Le, Loic Vial, Jibril Frej, Vincent Segonne, Maximin Coavoux, B. Lecouteux, A. Allauzen, B. Crabbé, L. Besacier, and Didier Schwab. 2020. Flaubert: Unsupervised language model pre-training for french. In $L R E C$.

H. Levesque, E. Davis, and L. Morgenstern. 2011. The winograd schema challenge. In $K R$.

Jindřich Libovický, Rudolf Rosa, and Alexander Fraser. 2019. How language-neutral is multilingual bert?

Yinhan Liu, Myle Ott, Naman Goyal, Jingfei Du, Mandar Joshi, Danqi Chen, Omer Levy, Mike Lewis, Luke Zettlemoyer, and Veselin Stoyanov. 2019. RoBERTa: A robustly optimized BERT pretraining approach. arXiv preprint arXiv:1907.11692.

Anthony McEnery, Paul Baker, Rob Gaizauskas, and Hamish Cunningham. 2000. Emille: Building a corpus of south asian languages. VIVEK-BOMBAY-, 13(3):22-28.

Yu Meng, Jiaxin Huang, Guangyuan Wang, Chao Zhang, Honglei Zhuang, Lance Kaplan, and Jiawei Han. 2019. Spherical text embedding. In Advances in Neural Information Processing Systems, pages 8206-8215.

Tomas Mikolov, Kai Chen, G. S. Corrado, and J. Dean. 2013a. Efficient estimation of word representations in vector space. CoRR, abs/1301.3781.

Tomas Mikolov, Ilya Sutskever, Kai Chen, Greg S Corrado, and Jeff Dean. 2013b. Distributed representations of words and phrases and their compositionality. In Advances in neural information processing systems, pages 3111-3119.

Sandeep Sricharan Mukku and Radhika Mamidi. 2017. ACTSA: Annotated corpus for Telugu sentiment analysis. In Proceedings of the First Workshop on Building Linguistically Generalizable NLP Systems, pages 54-58, Copenhagen, Denmark. Association for Computational Linguistics.

Pedro Javier Ortiz Suárez, Benoît Sagot, and Laurent Romary. 2019. Asynchronous Pipeline for Processing Huge Corpora on Medium to Low Resource Infrastructures. In 7th Workshop on the Challenges in the Management of Large Corpora (CMLC 7), Cardiff, United Kingdom. Leibniz-Institut für Deutsche Sprache.
Pedro Javier Ortiz Suarez, Benoît Sagot, and Laurent Romary. 2019. Asynchronous pipelines for processing huge corpora on medium to low resource infrastructures. In Proceedings of the Workshop on Challenges in the Management of Large Corpora, pages 9 - 16, Mannheim. Leibniz-Institut für Deutsche Sprache.

Xiaoman Pan, Boliang Zhang, Jonathan May, Joel Nothman, Kevin Knight, and Heng Ji. 2017. Crosslingual name tagging and linking for 282 languages. In Proceedings of the 55th Annual Meeting of the Association for Computational Linguistics (Volume 1: Long Papers), pages 1946-1958, Vancouver, Canada. Association for Computational Linguistics.

Jeffrey Pennington, Richard Socher, and Christopher Manning. 2014. Glove: Global vectors for word representation. In Proceedings of the 2014 conference on empirical methods in natural language processing $(E M N L P)$, pages 1532-1543.

Matthew Peters, Mark Neumann, Mohit Iyyer, Matt Gardner, Christopher Clark, Kenton Lee, and Luke Zettlemoyer. 2018. Deep contextualized word representations. In Proceedings of the 2018 Conference of the North American Chapter of the Association for Computational Linguistics: Human Language Technologies, Volume 1 (Long Papers), pages 22272237, New Orleans, Louisiana. Association for Computational Linguistics.

Fabio Petroni, Tim Rocktäschel, Sebastian Riedel, Patrick Lewis, Anton Bakhtin, Yuxiang $\mathrm{Wu}$, and Alexander Miller. 2019. Language models as knowledge bases? In Proceedings of the 2019 Conference on Empirical Methods in Natural Language Processing and the 9th International Joint Conference on Natural Language Processing (EMNLP-IJCNLP), pages 2463-2473, Hong Kong, China. Association for Computational Linguistics.

Telmo Pires, Eva Schlinger, and Dan Garrette. 2019. How multilingual is multilingual BERT? In Proceedings of the 57th Annual Meeting of the Association for Computational Linguistics, pages 49965001, Florence, Italy. Association for Computational Linguistics.

Alec Radford. 2018. Improving language understanding by generative pre-training. https://cdn.openai.com/research-covers/ language-unsupervised/language _ understanding_paper.pdf.

Alec Radford, Jeff Wu, Rewon Child, David Luan, Dario Amodei, and Ilya Sutskever. 2019. Language models are unsupervised multitask learners. https://cdn.openai.com/ better-language-models/language_models_are unsupervised_multitask_learners.pdf.

Colin Raffel, Noam Shazeer, Adam Roberts, Katherine Lee, Sharan Narang, Michael Matena, Yanqi Zhou, Wei Li, and Peter J. Liu. 2019. Exploring the limits 
of transfer learning with a unified text-to-text transformer. arXiv e-prints.

Nils Reimers and Iryna Gurevych. 2019. SentenceBERT: Sentence embeddings using Siamese BERTnetworks. In Proceedings of the 2019 Conference on Empirical Methods in Natural Language Processing and the 9th International Joint Conference on Natural Language Processing (EMNLP-IJCNLP), pages 3982-3992, Hong Kong, China. Association for Computational Linguistics.

Sebastian Ruder, Anders Søgaard, and Ivan Vulić. 2019. Unsupervised cross-lingual representation learning. In Proceedings of the 57th Annual Meeting of the Association for Computational Linguistics: Tutorial Abstracts, pages 31-38, Florence, Italy. Association for Computational Linguistics.

Shashank Siripragrada, Jerin Philip, Vinay P. Namboodiri, and C V Jawahar. 2020. A multilingual parallel corpora collection effort for Indian languages. In Proceedings of The 12th Language Resources and Evaluation Conference, pages 3743-3751, Marseille, France. European Language Resources Association.

Alex Wang, Yada Pruksachatkun, Nikita Nangia, Amanpreet Singh, Julian Michael, Felix Hill, Omer Levy, and Samuel Bowman. 2019. Superglue: A stickier benchmark for general-purpose language understanding systems. In $\mathrm{H}$. Wallach, $\mathrm{H}$. Larochelle, A. Beygelzimer, F. dAlché Buc, E. Fox, and R. Garnett, editors, Advances in Neural Information Processing Systems 32, pages 3266-3280. Curran Associates, Inc.

Alex Wang, Amanpreet Singh, Julian Michael, Felix Hill, Omer Levy, and Samuel Bowman. 2018. GLUE: A multi-task benchmark and analysis platform for natural language understanding. In Proceedings of the 2018 EMNLP Workshop BlackboxNLP: Analyzing and Interpreting Neural Networks for NLP, pages 353-355, Brussels, Belgium. Association for Computational Linguistics.

Guillaume Wenzek, Marie-Anne Lachaux, Alexis Conneau, Vishrav Chaudhary, Francisco Guzman, Armand Joulin, and Edouard Grave. 2019. Ccnet: Extracting high quality monolingual datasets from web crawl data. arXiv preprint arXiv:1911.00359.

Liang Xu, Xuanwei Zhang, Lu Li, Hai Hu, Chenjie Cao, Weitang Liu, Junyi Li, Yudong Li, Kai Sun, Yechen $\mathrm{Xu}$, et al. 2020. Clue: A chinese language understanding evaluation benchmark. arXiv preprint arXiv:2004.05986.

\section{A Publicly Available Datasets}

In this section, we summarize the publicly available datasets which are part of the IndicGLUE benchmark. The essential details of the datasets are described in Table 11. Except WNLI and

\begin{tabular}{|c|c|c|c|c|}
\hline \multirow[t]{2}{*}{ Lang } & \multirow[t]{2}{*}{ Dataset } & \multirow[t]{2}{*}{$\mathbf{N}$} & \multicolumn{2}{|c|}{ \# Examples } \\
\hline & & & Train & Test \\
\hline hi & BBC Articles ${ }^{10}$ & 6 & 3,467 & 866 \\
\hline bn & Soham Articles ${ }^{11}$ & 6 & 11,284 & 1411 \\
\hline $\mathrm{gu}$ & & 3 & 5,269 & 659 \\
\hline $\mathrm{ml}$ & iNLTK & 3 & 5,036 & 630 \\
\hline $\mathrm{mr}$ & Headlines $^{12}$ & 3 & 9,672 & 1,210 \\
\hline ta & & 3 & 5,346 & 669 \\
\hline \multirow[t]{2}{*}{ hi } & IITP+ Movie Reviews & 3 & 2,480 & 310 \\
\hline & IITP Product Reviews ${ }^{13}$ & 3 & 4,182 & 523 \\
\hline te & ACTSA corpus $^{14}$ & 3 & 4,328 & 541 \\
\hline hi & MIDAS Discourse Mode ${ }^{15}$ & 5 & 7974 & 997 \\
\hline hi & & 2 & 2500 & 900 \\
\hline pa & Amrita Paraphrase ${ }^{16}$ & 2 & 1700 & 500 \\
\hline ta & Subtask 1 & 2 & 2500 & 900 \\
\hline $\mathrm{ml}$ & & 2 & 2500 & 900 \\
\hline hi & & 2 & 3500 & 1400 \\
\hline pa & Amrita Paraphrase & 2 & 2200 & 750 \\
\hline ta & Subtask 2 & 2 & 3500 & 1400 \\
\hline $\mathrm{ml}$ & & 2 & 3500 & 1400 \\
\hline hi & COPA & 2 & 362 & 449 \\
\hline $\mathrm{gu}$ & (new, translated) & 2 & 362 & 448 \\
\hline $\mathrm{mr}$ & & 2 & 362 & 449 \\
\hline hi & WNLI & 2 & 636 & 147 \\
\hline $\mathrm{gu}$ & (new, translated) & 2 & 636 & 147 \\
\hline $\mathrm{mr}$ & & 2 & 636 & 147 \\
\hline
\end{tabular}

Table 11: IndicGLUE public datasets statistics. $\mathbf{N}$ is the number of classes.

COPA, all other datasets are publicly available. They cover sentiment analysis, new article classification, news headline classification, discourse mode classification. The WNLI and COPA datasets are manual translations of the original English datasets into a few Indian languages.

\section{Some notes on public datasets}

- The IITP+ Movie Reviews sentiment analysis dataset is created by merging IIT-Patna

\footnotetext{
${ }^{10} \mathrm{https}$ //github.com/NirantK/hindi2vec/releases/tag/bbchindi-v0.1

${ }^{11} \mathrm{https} / /$ www.kaggle.com/csoham/classification-bengalinews-articles-indicnlp

${ }^{12} \mathrm{https} / /$ github.com/goru001/inltk

${ }^{13} \mathrm{http}: / /$ www.iitp.ac.in/ ai-nlp-ml/resources.html

${ }^{14} \mathrm{https}: / /$ github.com/NirantK/bharatNLP/releases

${ }^{15} \mathrm{https}: / /$ github.com/midas-research/hindi-discourse

${ }^{16} \mathrm{http}: / /$ www.nlp.amrita.edu/dpil_cen
} 


\begin{tabular}{llrr}
\hline Lang & \multicolumn{1}{c}{ Classes } & \multicolumn{2}{c}{ \# Articles } \\
& & Train & Test \\
\hline pa & BIZ, ENT, POL, SPT & 2,496 & 312 \\
bn & ENT, SPT & 11,200 & 1,400 \\
or & BIZ, CRM, ENT, SPT & 17,750 & 2,250 \\
gu & BIZ, ENT, SPT & 1,632 & 204 \\
mr & ENT, STY, SPT & 3,600 & 450 \\
\hline kn & ENT, STY, SPT & 24,000 & 3,000 \\
te & ENT, BIZ, SPT & 19,200 & 2,400 \\
ml & BIZ, ENT, SPT, TECH & 4,800 & 600 \\
ta & ENT, POL, SPT & 7,200 & 900 \\
\hline
\end{tabular}

Table 12: IndicGLUE News category dataset statistics. The following are the categories: entertainment: ENT, sports: SPT, business: BIZ, lifestyle; STY, techology: TECH, politics: POL, crime: CRM.

dataset with the smaller IIT-Bombay and iNLTK datasets.

- The IIT-Patna Movie and Product review datasets have 4 classes namely postive, negative, neutral and conflict. We ignored the conflict class.

- In the Telugu-ACTSA corpus, we evaluated only on the news line dataset (named as telugu_sentiment_fasttext.txt) and ignored all the other domain datasets as they have very few data-points.

\section{B IndicGLUE News Category Dataset}

The IndicGLUE news category dataset is a collection of articles labeled with news categories. We used this dataset in the evaluation of word embeddings and language models. Table 12 provides the statistics of the dataset.

\section{IndicGLUE Datasets}

We provide some additional statistics for the $I n$ dic GLUE dataset in Table 2. In the following subsections, we show some examples of the datasets that we created .

\section{C.1 News Category Classification}

Article Snippet கர்நாடக சட்டப் பேரவையில் வெற்றி பெற்ற எம்எல்ஏக்கள் இன்று பதவியேற்றுக் கொண்ட நிலையில் , காங்கிரஸ் எம்எல்ஏ ஆனந்த் சிங் க்கள் ஆப்சென்ட ஆகி அதிர்ச்சியை ஏற்படுத்தியுள்ளார் 2ச்சநீதிமன்ற 2த்தரவுப்பட இன்று மாலை முதலமைச்ச் எடியரரப்பா இன்று நம்பிக்கை

\begin{tabular}{crrr}
\hline & Min & Max & Avg \\
\hline Headline Prediction & & & \\
Article Length (in words) & 12 & 448 & 154 \\
Headline Length (in words) & 2 & 47 & 8.9 \\
\hline $\begin{array}{c}\text { Wikipedia Section-Title Prediction } \\
\text { Section Length (in words) } \\
\text { Title Length (in words) }\end{array}$ & 1 & 9554 & 140 \\
\hline News Category Classification & & & \\
Article Length (in words) & 23 & 4649 & 205 \\
\hline Cloze-style QA & & & \\
Question Length (in words) & 7 & 190 & 63 \\
\hline Cross-lingual Sentence Retrieval & & & \\
Number of Sent Pairs per Lang Pair & 752 & 6463 & 4904 \\
\hline
\end{tabular}

Table 13: Additional IndicGLUE statistics.

வாக்கெடப்பு நடத்தி பெரீம்பான்மையை நிரூபிக்க 2ச்சநீதிமன்றம் 2த்தரவிட்டது .

Category: Politics

\section{C.2 Headline Prediction}

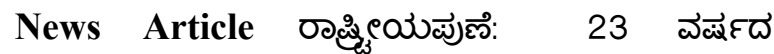

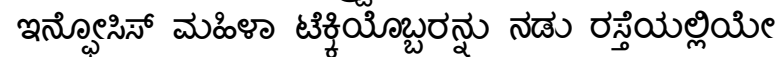

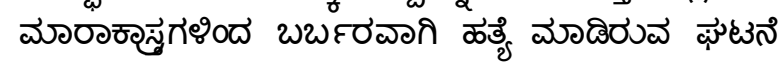

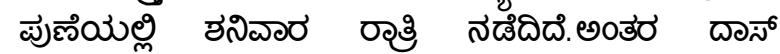

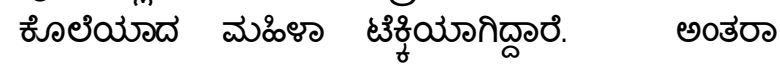

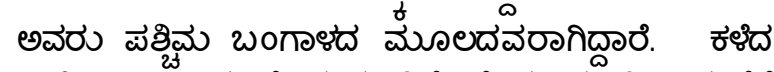
రవత్రి 8.00 గెంటి సుమోరిగి శాలస మొగిస మునిగి

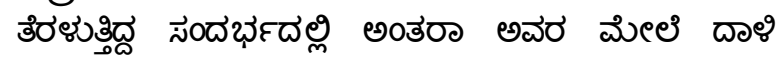

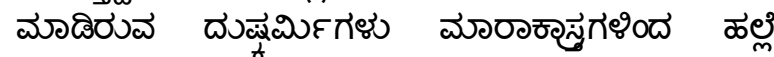

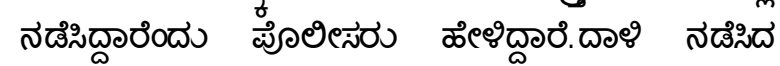

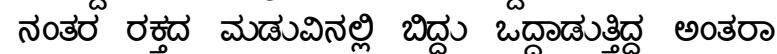

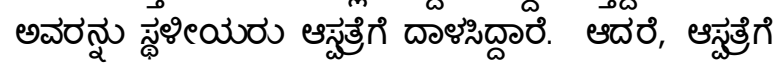

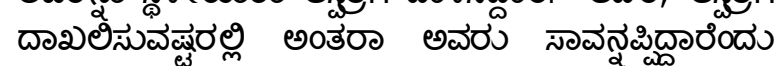

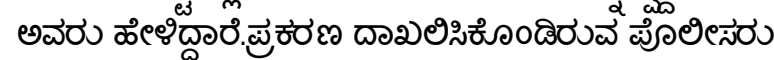
उనిఖ ఆరంభిసిద్దారి"

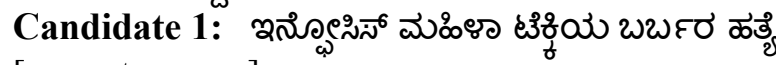
[correct answer]

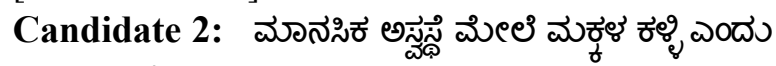

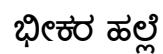

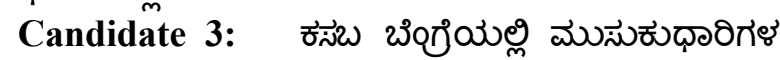

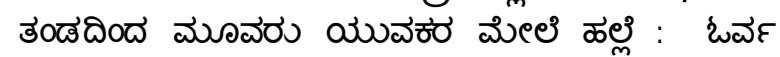
గెంభेలठ

Candidate 4: ซణిది రాజ్యదిల్లి mobile బందా,

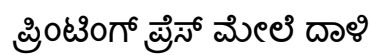

\section{C.3 Wikipedia Section Title Prediction}

Section Text 2005માં, જેકમેન નિર્માણ કંપની, સીડ પ્રોડકશન્સ ઊભી કરવા તેના લાંબાસમયના મદદનીશ જહોન પાલેર્મો સાથે જોડાયા, જેમનો પ્રથમ પ્રોજેકટ 2007માં વિવા લાફલિન હતો. જેકમેનની અભિનેત્રી પત્ની ડેબોરા-લી ફર્નેસ પણ કંપનીમાં જોડાઈ, અને પાલેર્મોએ 


\begin{tabular}{lrrr}
\hline \multicolumn{1}{c}{ Model } & Params & \multicolumn{2}{c}{ \#Train Tokens } \\
& & Total & Indic \\
\hline XLM-R & $125 \mathrm{M}$ & $295 \mathrm{~B}$ & $3.99 \mathrm{~B}$ \\
mBERT & $110 \mathrm{M}$ & $18.2 \mathrm{~B}^{*}$ & $184 \mathrm{M}^{*}$ \\
IndicBERT base & $12 \mathrm{M}$ & $8.93 \mathrm{~B}$ & $7.59 \mathrm{~B}$ \\
IndicBERT large & $18 \mathrm{M}$ & $8.93 \mathrm{~B}$ & $7.59 \mathrm{~B}$ \\
\hline
\end{tabular}

Table 14: Comparison of Different Models. *Estimated.

પોતાના, ફર્નેસ અને જેકમેન માટે " યુનિટી " અર્થવાળ। લખાણની આ ત્રણ વીંટીઓ બનાવી.[2৩] ત્રણેયના સહયોગ અંગે જેકમેને જણાવ્યું કे “ મારી જિંદગીમાં જેમની સાથે મેં કામ કર્યું તે ભાગીદારો અંગે ડેબ અને જહોન પાલેર્મો અંગે હું ખૂબ નસીબદાર છું. ખરેખર તેથી કામ થયું. અમારી પાસે જુદું જુદું સાર્મથ્ય હતું. હું તે પસંદ કરતો હતો. I love it.તે ખૂબ ઉત્તેજક છે. "[2૮]ફોકસ આધ્ધારિત સીડ લેબલ, આમન્ડા સ્કિવેઈટઝર, કેથરિન ટેન્બલિન, એલન મંડેલબમ અને જોય મરિનો તેમજ સાથે સિડની આધારિત નિર્માણ કચેરીનું સંચાલન કરનાર અલાના ફ્રીનો સમાવેશ થતાં કદમાં વિસ્તૃત બની. આ કંપીનોનો ઉछેશ જેકમેનના વતનના દેશની સ્થાનિક પ્રતિભાને કામે લેવા મધ્ચમ બજેટવાળી ફિલ્મો બનાવવાનો છે.

Candidate 1: એકસ-મેન

Candidate 2: કારકીર્દ

Candidate 3: નિર્માણ કંપન [correct answer]

Candidate 4: ઓસ્ટ્રેલિય

\section{C.4 Cloze-style Question Answering}

Question অক্টোবরফেস্ট হুলো দুই সপ্তাহের একটি অনুষ্ঠান, যা প্রতিবছর <MASK> অনুষ্ঠিত হয়। এটি মূলত সেপ্টেম্বর মাসের শেষ দিকে এবং অক্টোবর মাসের শুরুর দিকে পালন করা হয়ে থাকে। প্রতিবছর প্রায় ৬০লক্ষ লোক এই আয়োজনে অংশগ্রহণ করেন। জার্মানিতে এবং সারাবিশ্বে অক্টোবরফেস্ট নামে আরও উৎসব পালন করা হয়ে থাকে।

Candidate 1: মিউনিখে [correct answer]

Candidate 2: ভেনেজুয়েলাতে

Candidate 3: বার্লিনে

Candidate 4: শ্রীলঙ্কাতে

\section{C.5 Model Details}

Table 14 compares our models with existing pretrained models. 